\title{
Contents
}

\section{Three Sources and Three Component Parts of the Concept of Dissipative}

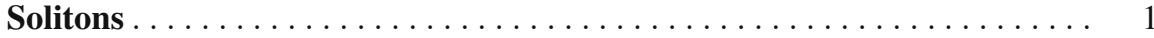

N. Akhmediev and A. Ankiewicz

$1 \quad$ Introduction . . . . . . . . . . . . . . . . . . . . . 1

2 Cubic-Quintic Complex Ginzburg-Landau Equation . .......... 7

3 The Method of Moments ........................ 8

$4 \quad$ Pulsating Solitons and Their Approximations $[37,38] \ldots \ldots \ldots$

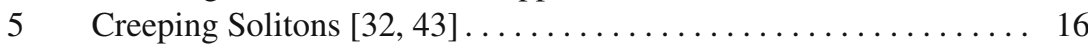

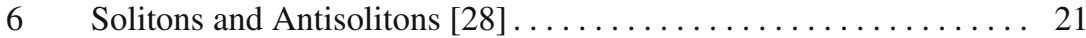

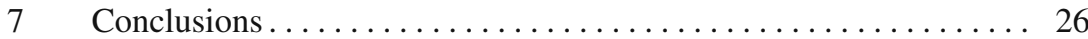

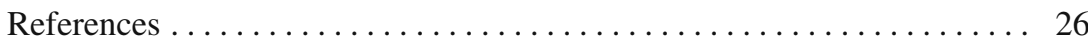

Solitons in Viscous Flows . . . . . . . . . . . . . . . . . . . . . . . . . . . . . . . . 29

M.G. Velarde and A.A. Nepomnyashchy

$1 \quad$ Introduction . . . . . . . . . . . . . . . . . . . 29

$2 \quad$ Basic Models ............................. 30

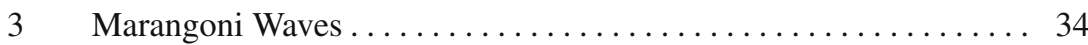

$4 \quad$ Waves in Flowing Films $\ldots \ldots \ldots \ldots \ldots \ldots \ldots \ldots \ldots \ldots \ldots \ldots$

5 Conclusions ............................... 48

References .............................. 48

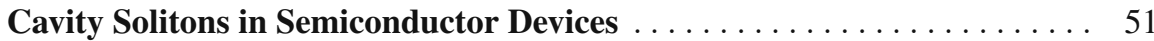

L.A. Lugiato, F. Prati, G. Tissoni, M. Brambilla, S. Barland, M. Giudici, and J.R. Tredicce

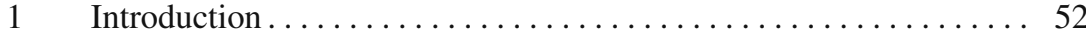

2 Cavity Solitons: Properties and Applicative Features . . . . . . . . . 54

3 Cavity Solitons in Driven VCSELs ................. 60

$4 \quad$ VCSELs with Saturable Absorber: The Cavity Soliton Laser . . . . . 84

5 Conclusions and Perspectives ...................... 89

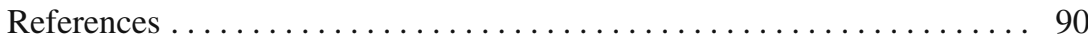


Dissipative Solitons in Laser Systems

with Non-local and Non-instantaneous Nonlinearity $\ldots \ldots \ldots \ldots \ldots$

N.N. Rosanov, S.V. Fedorov, and A.N. Shatsev

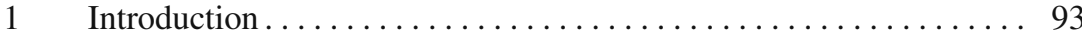

2 The Model and Initial Equations . . . . . . . . . . . . . . 95

3 Transversely Homogeneous Lasing . . . . . . . . . . . . . . 96

$4 \quad$ Single Motionless Dissipative Solitons and Their Complexes . . . . . 98

5 Bifurcation from Motionless to Slowly Moving Structures . . . . . . . 102

6 Slowly Moving Dissipative Solitons . . . . . . . . . . . . . 105

7 Flying and Non-stationary Dissipative Solitons . . . . . . . . 106

8 Conclusions ............................... 108

References .................................. 111

Excitability Mediated by Dissipative Solitons in Nonlinear Optical

Cavities .

P. Colet, D. Gomila, A. Jacobo, and M.A. Matías

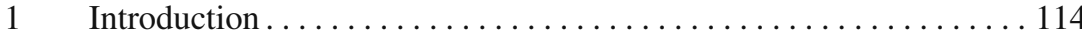

$2 \quad$ Model................................... 115

3 Dissipative Solitons for Homogeneous Pump . . . . . . . . . . 116

$4 \quad$ Azimuthal Instabilities . . . . . . . . . . . . . . . . . . . . . . . 119

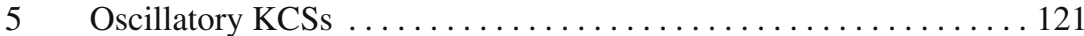

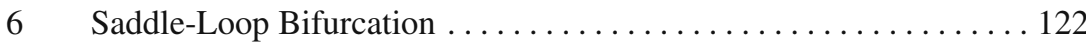

7 Quantitative Phase Space Projection .................. 124

$8 \quad$ Excitable Behavior ............................ 127

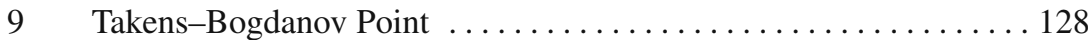

10 Effect of an Addressing Gaussian Beam . . . . . . . . . . . 130

11 Concluding Remarks . . . . . . . . . . . . . . . . . . 133

References .................................... 134

Temporal Soliton "Molecules" in Mode-Locked Lasers: Collisions,

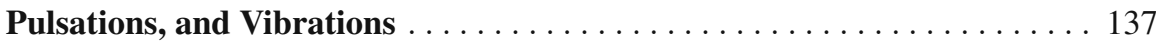

P. Grelu and J.M. Soto-Crespo

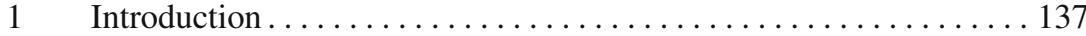

2 Collisions Between Dissipative Solitons Inside a Laser Cavity . . . . 140

3 Pulsations of Dissipative Solitons . . . . . . . . . . . . . . . 153

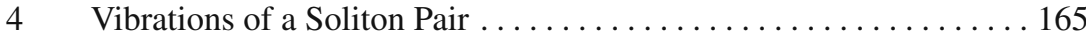

$5 \quad$ Conclusion and Prospects ...................... 170

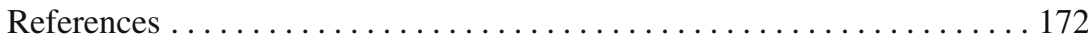

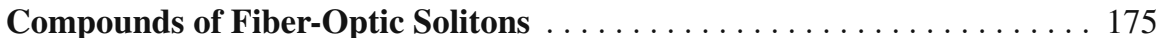

F. Mitschke

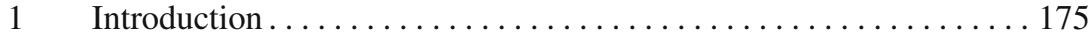

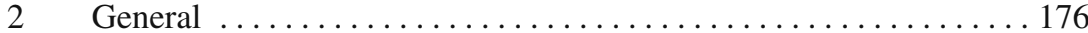

3 Soliton Ensembles in a Feedback System . . . . . . . . . . . . 178

4 Soliton Molecules: Bound States in Dispersion-Managed Fiber . . . . 182

5 Bound States of Dark Solitons in Dispersion-Managed Fiber . . . . . 188 
6 Discussion and Conclusion ........................... 192

References ....................................... 193

Dissipative Nonlinear Structures in Fiber Optics . . . . . . . . . . . . . 195

S.K. Turitsyn and S. Boscolo

$1 \quad$ Introduction ................................. 195

2 Dissipative Solitons in High-Speed Fiber Communication Systems . 197

3 Self-Similar Parabolic Pulses in Active Fibers ................ 205

4 Application of Parabolic Pulses to Optical Signal Processing . . . . . 213

5 Conclusion ................................... 217

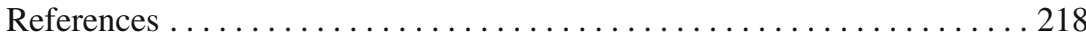

Three-Wave Dissipative Brillouin Solitons . . . . . . . . . . . . . . 221

C. Montes

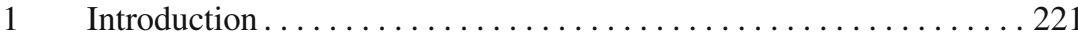

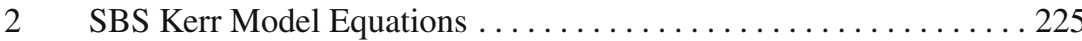

3 Three-Wave Dissipative Model ....................... 227

4 Asymptotic Three-Wave Dynamics in the Ring Cavity .......... 230

5 Backward-Traveling Three-Wave Solutions ................ 236

6 Dissipative Symmetric Three-Wave Solution ................. 237

7 Asymmetric Three-Wave Dissipative Solitons . . . . . . . . . . . . 242

8 Stability Criterion: Asymptotic Procedure ................ 245

$9 \quad$ Brillouin Fiber-Ring Laser Pulses . .................... 248

10 Systematic Experimental and Numerical Exploration of the Whole Soliton Localization Domain ................ 251

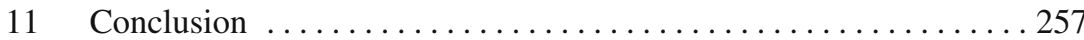

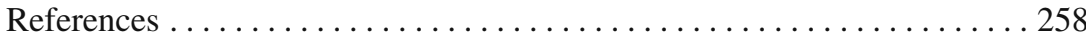

Spatial Dissipative Solitons Under Convective and Absolute Instabilities in Optical Parametric Oscillators . . . . . . . . . . . . . . . . . . . . 261

S. Coulibaly, C. Durniak, and M. Taki

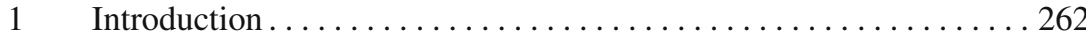

2 Convective Versus Absolute Instabilities in OPOs .............. 264

3 Degenerate Optical Parametric Oscillators ................. 269

4 Walk-Off Nonlinear Effects in Parametric Optical Oscillators . . ... 277

$5 \quad$ Concluding Remarks ............................. 285

References ..................................... 285

Discrete Breathers with Dissipation . . . . . . . . . . . . . . . . . . 289

S. Flach and A.V. Gorbach

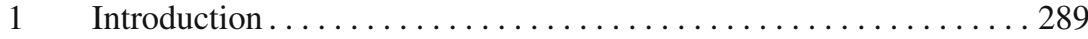

2 Basic Properties of Discrete Breathers ................... 296

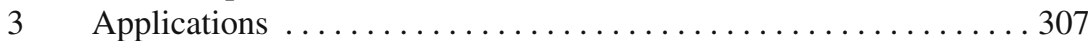

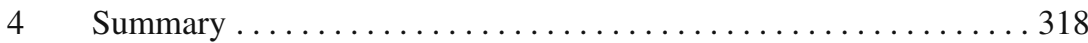

References ........................................... 319 
Anharmonic Oscillations, Dissipative Solitons and Non-Ohmic

Supersonic Electric Transport .

M.G. Velarde, W. Ebeling, and A.P. Chetverikov

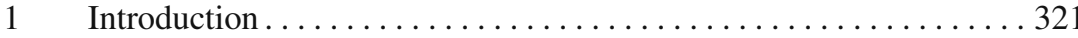

2 Solitons and Cnoidal Waves in a Toda Lattice ............ 323

3 Self-Organization with an Input-Output Energy Balance . . . . . . . 325

4 Electromechanical Toda Lattice . . . . . . . . . . . . . . . 327

5 From Ohm's Law to a Soliton-Mediated Supersonic Current . . . . . 330

$6 \quad$ Final Remarks . . . . . . . . . . . . . . . . . . . . . . . . . . . . . . 333

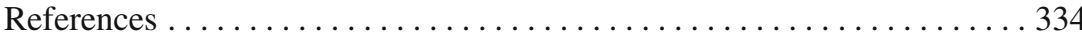

Coherent Optical Pulse Dynamics

in Nano-composite Plasmonic Bragg Gratings . . . . . . . . . . . . . . . 337

I.R. Gabitov, A.O. Korotkevich, A.I. Maimistov, and J.B. McMahon

$1 \quad$ Introduction . . . . . . . . . . . . . . . . . . . . . . . . 337

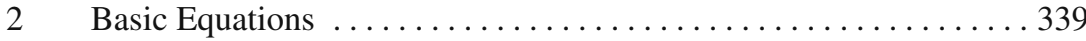

3 Solitary Wave Solutions . . . . . . . . . . . . . . . . . . 351

$4 \quad$ Energy Partition ............................... 354

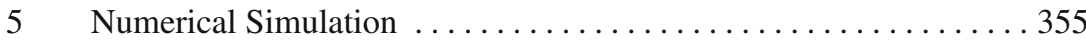

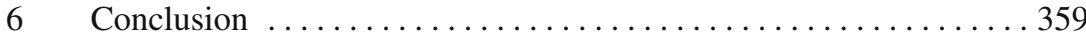

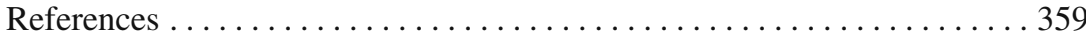

Collective Focusing and Modulational Instability of Light and Cold

Atoms . . . . . . . . . . . . . . . . . . . . . . . . . . . . . . . . . 361

M. Saffman and Y. Wang

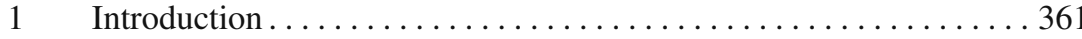

2 Dynamical Equations . . . . . . . . . . . . . . . . . . . 362

3 Coupled Optical and Atomic Solitons ................. 365

4 Two-Beam Coupling and Atomic Density Perturbations . . . . . . . . 367

5 Modulational Instability . . . . . . . . . . . . . . . . . . . . . . . 372

6 Modulational Instability with Density Changes . . . . . . . . . . . 374

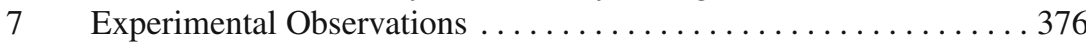

8 Conclusion ................................... 379

References .................................... 380

On Vegetation Clustering, Localized Bare Soil Spots and Fairy Circles . . 381 M. Tlidi, R. Lefever, and A. Vladimirov

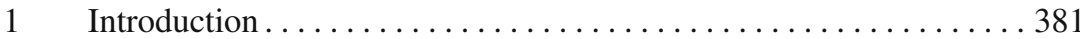

2 Mean-Field Model of Vegetation Evolution . . . . . . . . . . . . . . 384

3 Weak-Gradient Approximation . . . . . . . . . . . . . . . . . . . . . . . 387

$4 \quad$ Clustering and Periodic Vegetation Patterns ................ 390

$5 \quad$ Pinning and Localized Bare Soil Spots . . . . . . . . . . . . . . 391

6 Attractive/Repulsive Interactions Between Bare Spots . . . . . . . . . 395

$7 \quad$ Conclusions . . . . . . . . . . . . . . . . . . . . . . . . . . . . 400

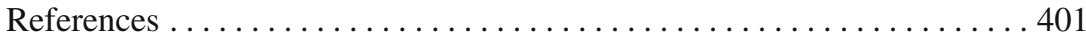


Propagation of Traveling Pulses in Cortical Networks $\ldots \ldots \ldots \ldots \ldots 3$

D. Golomb

1 Introduction

2 Propagating Pulses in Networks of Excitatory Neurons . . . . . . . . 405

3 Propagating Pulses in Networks of Excitatory

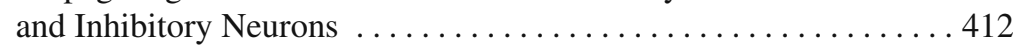

4 Discussion ................................ 426

References ........................................ 429

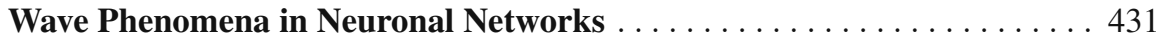
W.C. Troy

1 Introduction . . . . . . . . . . . . . . . . . . . . . 431

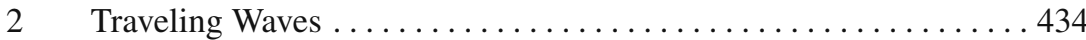

3 Wave Fronts ............................. 435

4 Solitary Traveling Waves ...................... 438

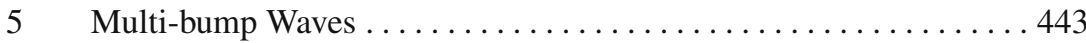

6 Two Dimensions . . . . . . . . . . . . . . . . . . . . . . . . . . . 444

$7 \quad$ Conclusions . . . . . . . . . . . . . . . . . . . . . . . . . . 450

References ............................... 451

Spiral Waves and Dissipative Solitons in Weakly Excitable Media . . . . . . 453 V.S. Zykov

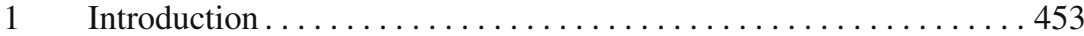

2 Excitable Medium Model . . . . . . . . . . . . . . . . . . . . . 455

3 Free Boundary Problem for Stabilized Wave Segments . . . . . . . . . 457

4 Wave Spots Rotating Along a Disk Boundary . . . . . . . . . . . . . 462

5 Spiral Waves Rotating Around a Disk Center . . . . . . . . . . . . . . . 466

6 Spiral Waves in an Unbounded Medium . . . . . . . . . . . . . . 469

$7 \quad$ Discussion ............................ 471

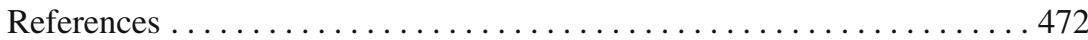

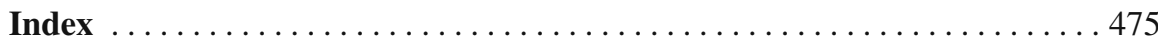

